\title{
Macro- and Microstructural Changes in Patients with Spinocerebellar Ataxia Type 6: Assessment of Phylogenetic Subdivisions of the Cerebellum and the Brain Stem
}

\author{
K. Sato, K. Ishigame, S.H. Ying, K. Oishi, M.I. Miller, and S. Mori
}

\begin{abstract}
BACKGROUND AND PURPOSE: Site-specific degeneration patterns of the infratentorial brain in relation to phylogenetic origins may relate to symptoms in patients with spinocerebellar degeneration, but the patterns are still unclear. We investigated macro- and microstructural changes of the infratentorial brain based on phylogenetic origins and their correlation with symptoms in patients with spinocerebellar ataxia type 6.
\end{abstract}

MATERIALS AND METHODS: MR images of 9 patients with spinocerebellar ataxia type 6 and 9 age- and sex-matched controls were obtained. We divided the infratentorial brain on the basis of phylogenetic origins and performed an atlas-based analysis. Comparisons of the 2 groups and a correlation analysis assessed with the International Cooperative Ataxia Rating Scale excluding age effects were performed.

RESULTS: A significant decrease of fractional volume and an increase of mean diffusivity were seen in all subdivisions of the cerebellum and in all the cerebellar peduncles except mean diffusivity in the inferior cerebellar peduncle in patients compared with controls $(P<.0001$ to $<.05$ ). The bilateral anterior lobes showed the strongest atrophy. Fractional volume decreased mainly in old regions, whereas mean diffusivity increased mainly in new regions of the cerebellum. Reflecting this tendency, the International Cooperative Ataxia Rating Scale total score showed strong correlations in fractional volume in the right flocculonodular lobe and the bilateral deep structures and in mean diffusivity in the bilateral posterior lobes ( $r=0.73$ to \pm 0.87 ).

CONCLUSIONS: We found characteristic macro- and microstructural changes, depending on phylogenetic regions of the infratentorial brain, that strongly correlated with clinical symptoms in patients with spinocerebellar ataxia type 6 .

ABBREVIATIONS: FA = fractional anisotropy; ICARS = International Cooperative Ataxia Rating Scale; ICP = inferior cerebellar peduncle; LDDMM = large deformation diffeomorphic metric mapping; $\mathrm{MCP}=$ middle cerebellar peduncle; $\mathrm{MD}=$ mean diffusivity; SCA6 = spinocerebellar ataxia type 6 ; SCP $=$ superior cerebellar peduncle

S pinocerebellar ataxia type six (SCA6) is a rare, late-onset, autosomal dominant disease. SCA6 is associated with a genetic defect ( $C A G$ repeat expansion) in the gene encoding for a subunit of a voltage-gated calcium channel, which is associated with neuronal loss mainly in Purkinje cells in the cerebellar cor-

Received May 8, 2014; accepted after revision July 14.

From the Russell H. Morgan Department of Radiology and Radiological Science (K.S., K.I., K.O., S.M.), Departments of Radiology (S.H.Y.), Neurology (S.H.Y.), and Ophthalmology (S.H.Y.), and Center for Imaging Science (M.I.M.), Johns Hopkins University School of Medicine, Baltimore, Maryland; Department of Radiology (K.S.), Juntendo University School of Medicine, Tokyo, Japan; Department of Radiology (K.I.), University of Yamanashi, Yamanashi, Japan; and F.M. Kirby Research Center for Functional Brain Imaging (S.M.), Kennedy Krieger Institute, Baltimore, Maryland.

K. Sato and K. Ishigame contributed equally to this work.

Please address correspondence to Kanako Sato, MD, PhD, Department of Radiology, Juntendo University School of Medicine, 2-1-1 Hongo, Bunkyo-ku, Tokyo, Japan 113-8421; e-mail: satokana.tky@gmail.com

- Indicates open access to non-subscribers at www.ajnr.org

http://dx.doi.org/10.3174/ajnr.A4085 tex. ${ }^{1}$ SCA6 is not fatal, but the accompanying cerebellar dysfunction is permanent and progressive. ${ }^{2}$ Cerebellar atrophy is a characteristic imaging feature, and mild atrophy of the brain stem and subtle atrophy of the thalamus or the cerebral cortex can occur. ${ }^{3,4}$

Although the definite diagnosis of SCA6 is made by genetic examination, MR imaging can narrow the differential diagnosis of neurodegenerative diseases that can cause ataxia. MR imaging helps select the candidates for genetic examination. ${ }^{5}$ Degeneration patterns of the infratentorial brain are the key to differentiating SCA6 from other neurodegenerative diseases presenting with ataxia, and cerebellar degeneration is a characteristic imaging feature in patients with SCA6. However, few studies ${ }^{6,7}$ have investigated site-specific degeneration patterns of the infratentorial brain, and their segmentations were not easy to assess clinically. Moreover, correlations between site-specific degeneration patterns and clinical symptoms require further characterization.

The cerebellum has 3 regions derived from different phyloge- 
netic origins (the archicerebellum, the paleocerebellum, and the neocerebellum $)^{8-10}$ that form distinct neural pathways between the cerebellum and other regions of the brain. These 3 regions are believed to have different functions. ${ }^{9,11,12}$ It is of great interest to know how the cerebellar macro- and microstructural changes in each phylogenetic region manifest as symptoms in patients with SCA6.

Thus, in the present study, we investigated changes of both volume and diffusion metrics: mean diffusivity (MD) and fractional anisotropy (FA) obtained with DTI. Changes of diffusion metrics have been reported in other types of spinocerebellar ataxia and in multiple system atrophy. ${ }^{13,14}$ There are few DTI studies of patients with SCA6, ${ }^{15,16}$ and they investigated only part of the cerebellum or the cerebellar peduncles with manual methods.

We hypothesized that clinical symptoms in patients with SCA6 are correlated with macro- and microstructural changes depending on the phylogenetic origins of the cerebellum. The purposes of this study were to investigate these correlations and to identify the site-specific degeneration patterns of the infratentorial brain that are also clinically assessable in patients with SCA6. To our knowledge, this is the first MR imaging study combining volumetric and DTI analyses based on phylogenetic regions.

\section{MATERIALS AND METHODS \\ Subjects}

This study was approved by our institutional review board and complied with the Health Insurance Portability and Accountability Act. Written informed consent was obtained from all participants. We acquired MR images of the brain in 9 patients with SCA6 ( 5 women and 4 men; mean age, $57.9 \pm 7.25$ years) and 9 age-matched healthy controls ( 5 women and 4 men; mean age, $58.8 \pm 7.51$ years) obtained between August 2005 and September 2009 from the Johns Hopkins University School of Medicine, and the images were retrospectively analyzed.

\section{Clinical Evaluation}

All participants completed a medical questionnaire, and the possibility of secondary ataxia was excluded. Symptoms were assessed with the International Cooperative Ataxia Rating Scale (ICARS). ${ }^{17}$ ICARS scores have a maximum of 100 points as the total of 4 subscores: the static score, limb score, dysarthria score, and oculomotor score.

The neurologic examinations of all of patients and controls were performed by a neurologist (S.H.Y.) at the departments of radiology, neurology, and ophthalmology at the Johns Hopkins University School of Medicine. The neurologist had $>10$ years of experience in the design and measurement of quantitative neurologic assessments and was blinded to the MR imaging results.

\section{Data Acquisition}

Brain imaging was performed with a $3 \mathrm{~T}$ MR imaging scanner (Intera; Philips Healthcare, Best, the Netherlands). An axial single-shot spin-echo echo-planar imaging sequence was used for the DTI. The parameters used were as follows: TR/TE, 6491/69 $\mathrm{ms}$; b-value, $700 \mathrm{~s} / \mathrm{mm}^{2}$; diffusion-encoding gradients, 30 directions; ${ }^{18}$ number of signals acquired, 2; FOV, $212 \times 212 \mathrm{~mm}$; matrix size, $256 \times 256 \mathrm{~mm}$; intersection gap, $0 \mathrm{~mm}$; section thick- ness, $2.2 \mathrm{~mm}$; section number, 65. EPI with no diffusion weighting $\left(b=0 \mathrm{~s} / \mathrm{mm}^{2}\right)$ was performed for use in spatial normalization.

3D-magnetization prepared rapid acquisition of gradient echo images and T2WI were acquired. T2WI was used to exclude other brain diseases. The MPRAGE parameters were as follows: TR/TE, 10.33/6 ms; FOV, $212 \times 212 \mathrm{~mm}$; matrix size, $256 \times 256$ $\mathrm{mm}$; intersection gap, $0 \mathrm{~mm}$; section thickness, $1.1 \mathrm{~mm}$; section number, 65. The T2WI parameters were the same as those for MPRAGE except for the following: TR/TE1/TE2, 3465/28.2/80.0 ms; section thickness, $1.5 \mathrm{~mm}$.

\section{Registration and Normalization}

We used a single-subject template, the Johns Hopkins UniversityMontreal Neurological Institute template, which was normalized to Montreal Neurological Institute space. ${ }^{19,20}$ Each participant's MPRAGE and DTI (FA, trace) images were skull-stripped by using statistical parametric mapping followed by manual delineation and were normalized to the templates by using a 9-parameter linear algorithm ${ }^{21}$ and large deformation diffeomorphic metric mapping (LDDMM) (http://www.birncommunity.org/toolscatalog/large-deformation-diffeomorphic-metric-mappinglddmm/). ${ }^{2,23}$ Using the deformation fields from LDDMM and the inverse linear matrix, we then warped our parcellation map (ie, Johns Hopkins University-Montreal Neurological Institutebased parcellation map) from the template to each subject's space. The details of the LDDMM operation are described elsewhere. $^{20,24}$ All image transformation was achieved with the software DiffeoMap (https://www.mristudio.org/).

\section{Creation of the Infratentorial Parcellation Map}

We modified the Johns Hopkins University-Montreal Neurological Institute-based parcellation map in which the original left and right cerebellum were manually parcellated, and we divided the cerebellum into 12 subdivisions to create the infratentorial parcellation map as shown in Fig 1. The vermis was separated from the hemispheres by its elevated edge and was subdivided into the anterior and the posterior vermis by the primary fissure. The left and right hemispheres were subdivided into the anterior and posterior lobes by the primary fissure. The tonsils were separated from the hemispheres by the secondary fissure.

The flocculonodular lobes were separated from the hemispheres and the tonsils by the posterolateral fissure. The deep structures were separated on the basis of the T1-weighted contrast of the Johns Hopkins University-Montreal Neurological Institute atlas. These included the white matter and the 3 nuclei (dentate, interpositus, and fastigial) of the corpus medullare with the exclusion of the middle cerebellar peduncle (MCP). We divided the brain stem into 6 subdivisions; the midbrain, the pons, the medulla, the superior cerebellar peduncle (SCP), the MCP, and the inferior cerebellar peduncle (ICP) following the original Johns Hopkins University-Montreal Neurological Institute-based parcellation map. In total, the infratentorial parcellation map had 18 subdivisions.

\section{Atlas-Based Analysis}

We conducted an atlas-based analysis by using the infratentorial parcellation map. We measured the volume, MD, and FA of each 


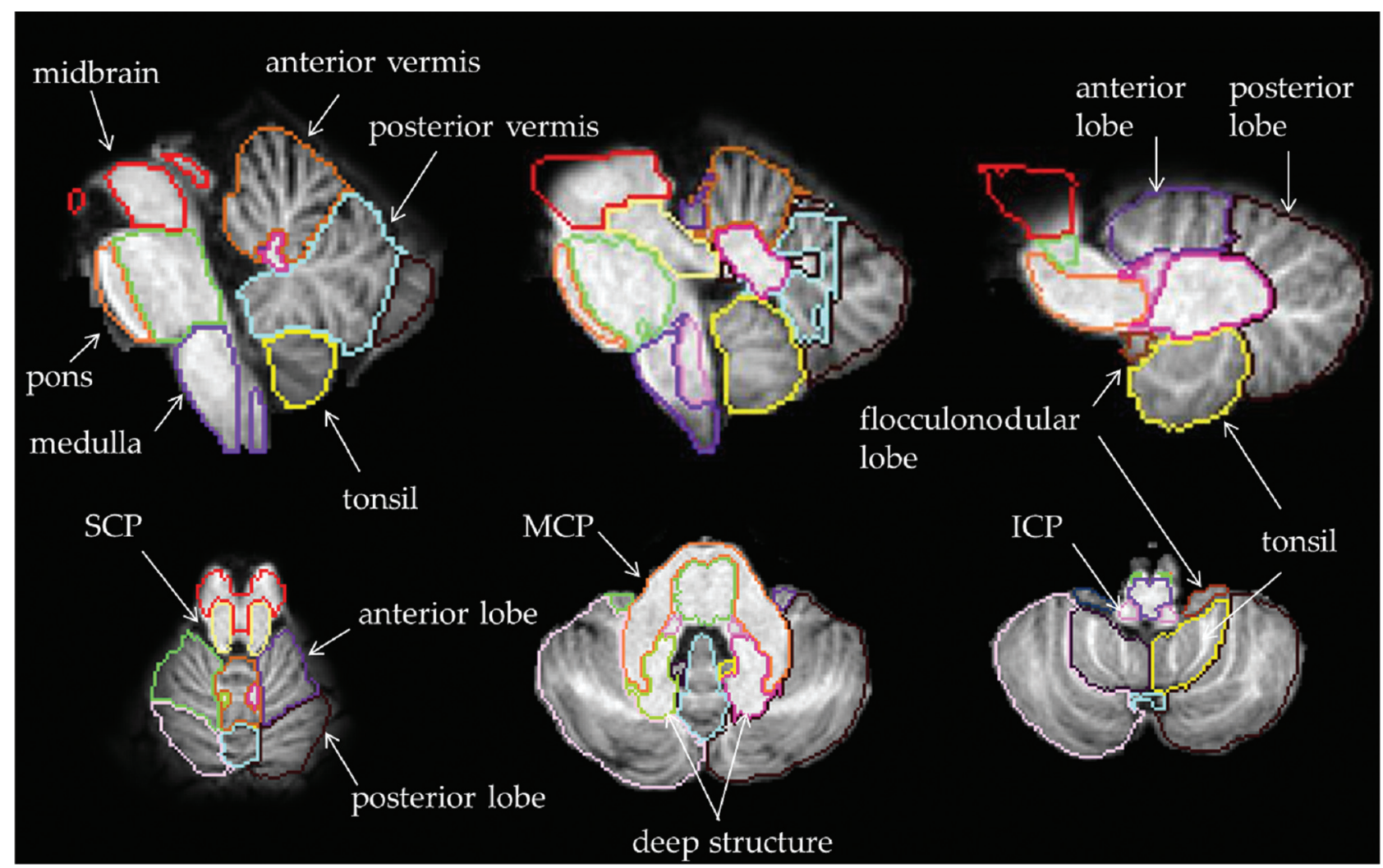

FIG 1. Infratentorial parcellation map. The cerebellum is divided into 12 subdivisions, and the brain stem, into 6 subdivisions.

subdivision and determined the correlation of each with the ICARS in each subdivision. Volume was evaluated as fractional volume (ie, the volume in each subdivision divided by the intracranial volume of each subject). We also calculated the percentage changes in the fractional volume, MD, and FA compared with controls by dividing each patient's data by the average of the controls' data in each subdivision.

CSF spaces were excluded by a signal-intensity threshold at 25 on MPRAGE images and by an MD threshold at $0.0023 \mathrm{~mm}^{2} / \mathrm{s}$ on DTI $\left(0.007 \mathrm{~mm}^{2} / \mathrm{s}\right.$ on trace images $)$. We investigated the correlation of fractional volume and MD in each subdivision to determine the partial volume effects of CSF due to atrophy on the results of MD.

\section{Statistics}

Statistical analyses were performed with the software SPSS, Version 13.0 (IBM, Armonk, New York). Comparisons of the patient and control groups were performed by using an independentsamples $t$ test after confirming normal distribution of the data by Kolmogorov-Smirnov testing. The Mann-Whitney $U$ test was used for the subdivisions in which the values were not normally distributed. Correlations between the data and the ICARS scores were assessed by using a Spearman correlation analysis. We conducted a multiple linear regression analysis with age effects as a covariant in the subdivisions in which the data were significantly correlated with age. A Bonferroni correction was adopted when the number of groups was $<5$, and the false discovery rate correction was adopted when the number of groups was $>5$.

\section{RESULTS}

\section{Whole Brain and the ICARS}

We first analyzed the data in 3 regions of the brain: the cerebrum, the cerebellum, and the brain stem. Among these, only the cerebellum showed a significant decrease in fractional volume and a significant increase in MD in the patients with SCA6 compared with the controls $(P<.0001)$. The FA did not show any significant differences. The fractional volume in both the cerebellum and the brain stem showed significant correlations with the ICARS total score $(P<.05, r=-0.80,-0.77)$. FA showed neither significant changes nor significant correlations.

\section{Infratentorial Subdivisions and the ICARS}

We next investigated changes of the data in the infratentorial subdivisions (Fig 2) and the correlations with the ICARS scores (Table). In the cerebellum, all subdivisions showed significant decreases of fractional volume $(P<.0001$ to $<.01)$ and increases of $\operatorname{MD}(P<.0001$ to $<.001)$ in patients with SCA6 compared with controls. FA showed significant decreases only in the bilateral posterior lobes and the right tonsil $(P<.01$ to $<.05)$. The strongest correlation with the ICARS total score was seen in the fractional volume in the right deep structure $(P<.01, r=-0.87)$ and the MD in the left posterior lobe $(P<.01, r=0.87)$.

Among the ICARS subscores, the static and dysarthria scores showed significant correlations mainly in old regions, including the archicerebellum and the spinocerebellum $(P<.001$ to $<.05$, $r=-.92$ to 0.85$)$. The limb score showed significant correlations mainly in the neocerebellum and the right deep structure $(P<$ $.05, r=-0.74$ to 0.73$)$. The strongest correlation with ICARS 

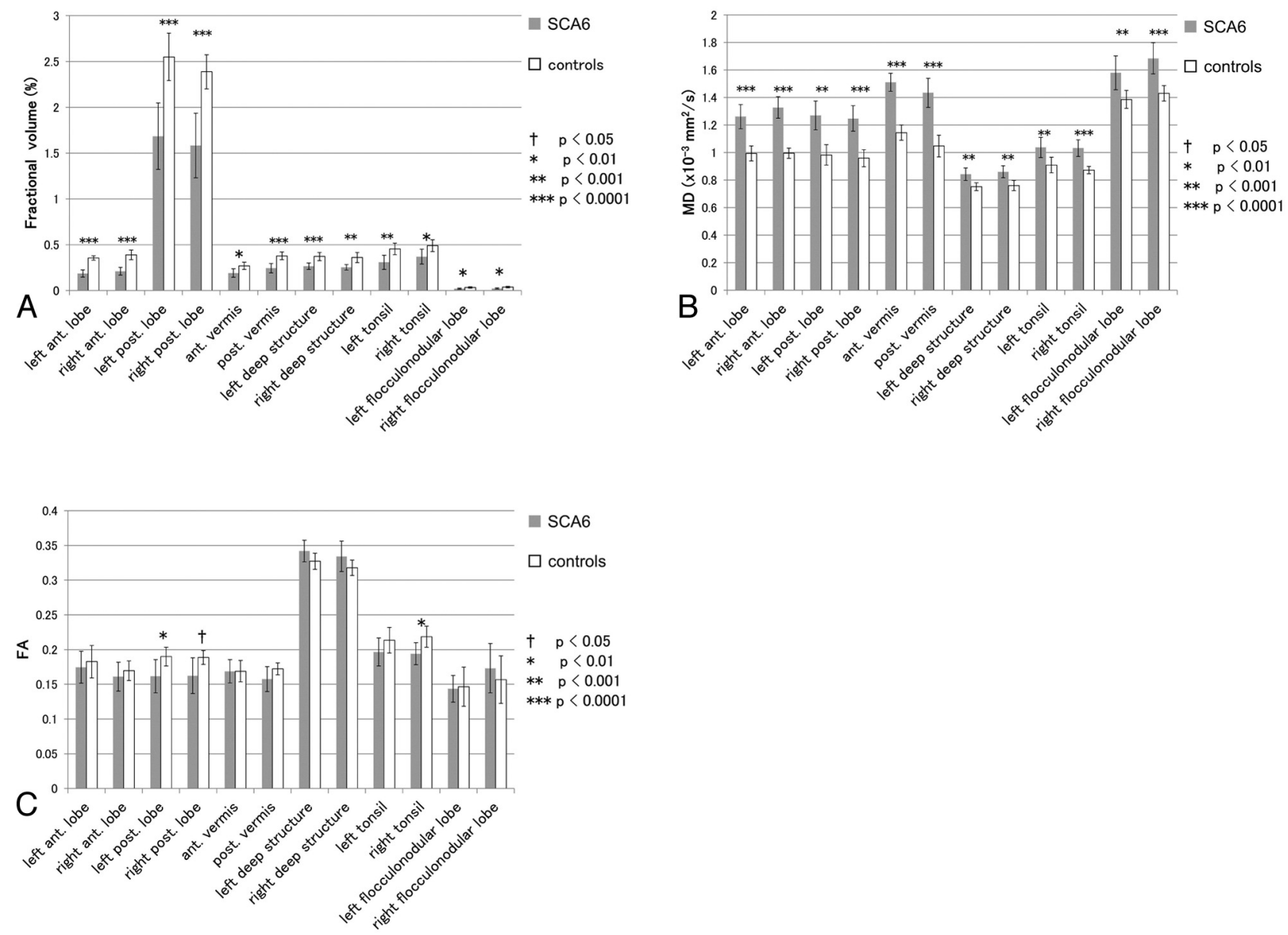

FIG 2. Comparison of the data in each cerebellar subdivision between patients with SCA6 and controls. Independent samples $t$ tests were performed with false discovery rate correction applied to $P<.05$. Ant. indicates anterior; post., posterior.

Correlations between the data and ICARS scores in the infratentorial brain ${ }^{\mathrm{a}}$

\begin{tabular}{|c|c|c|c|}
\hline SCA6 & Fractional Volume (\%) & $\mathrm{MD}\left(\times 10^{-3} \mathrm{~mm}^{2} / \mathrm{s}\right)$ & FA \\
\hline Left anterior lobe & Static $^{\mathrm{b}}(r=-0.82)$, Dysarthria $^{\mathrm{C}}(r=-0.73)$ & NS & NS \\
\hline Right anterior lobe & NS & NS & NS \\
\hline Left posterior lobe & NS & Total $^{\mathrm{d}}(r=0.87)$, Dysarthria $^{c}(r=0.77)$ & $\operatorname{Limb}^{c}(r=-0.73)$ \\
\hline Right posterior lobe & $\operatorname{Static}^{\mathrm{C}}(r=-0.66)$ & $\operatorname{Total}^{\mathrm{d}}(r=0.83), \operatorname{Limb}^{\mathrm{c}}(r=0.73)$ & NS \\
\hline Anterior vermis & NS & NS & NS \\
\hline Posterior vermis & NS & NS & $\operatorname{Limb}^{c}(r=-0.73)$ \\
\hline Left deep structure & $\operatorname{Total}^{c}(r=-0.75)$ & NS & NS \\
\hline Right deep structure & Total $^{\mathrm{d}}(r=-0.87), \operatorname{Limb}^{\mathrm{c}}(r=-0.74)$ & NS & NS \\
\hline Left tonsil & NS & NS & NS \\
\hline Right tonsil & NS & $\begin{array}{l}\text { Total }^{\mathrm{c}}(r=0.73), \text { Static }^{\mathrm{d}}(r=0.82), \\
\text { Dysarthria }^{\mathrm{d}}(r=0.85)\end{array}$ & NS \\
\hline Left flocculonodular lobe & $\operatorname{Static}^{c}(r=-0.74)$ & NS & NS \\
\hline Right flocculonodular lobe & $\begin{array}{l}\text { Total }^{\mathrm{d}}(r=-0.77), \text { Static }^{\mathrm{d}}(r=-0.92) \\
\quad \text { Dysarthria }^{\mathrm{d}}(r=-0.80)\end{array}$ & NS & NS \\
\hline Midbrain & $\operatorname{Total}^{\mathrm{C}}(r=-0.77)$ & NS & NS \\
\hline Pons & NS & NS & NS \\
\hline Medulla & NS & NS & NS \\
\hline SCP & $\operatorname{Static}^{c}(r=-0.71)$ & NS & NS \\
\hline MCP & NS & NS & NS \\
\hline ICP & NS & NS & NS \\
\hline
\end{tabular}

Note:-NS indicates not significant.

a ICARS scores that significantly correlated with the data are shown. "Static," "Dysarthria," and "Limb" are subscores of ICARS. A Spearman correlation analysis with false discovery rate correction applied to $P<.05$ was performed. A multiple linear regression analysis excluding age effects was performed in the subdivisions in which the data significantly correlated with age.

$\mathrm{b} P<.001$.

c $P<.05$.

d $P<.01$. 


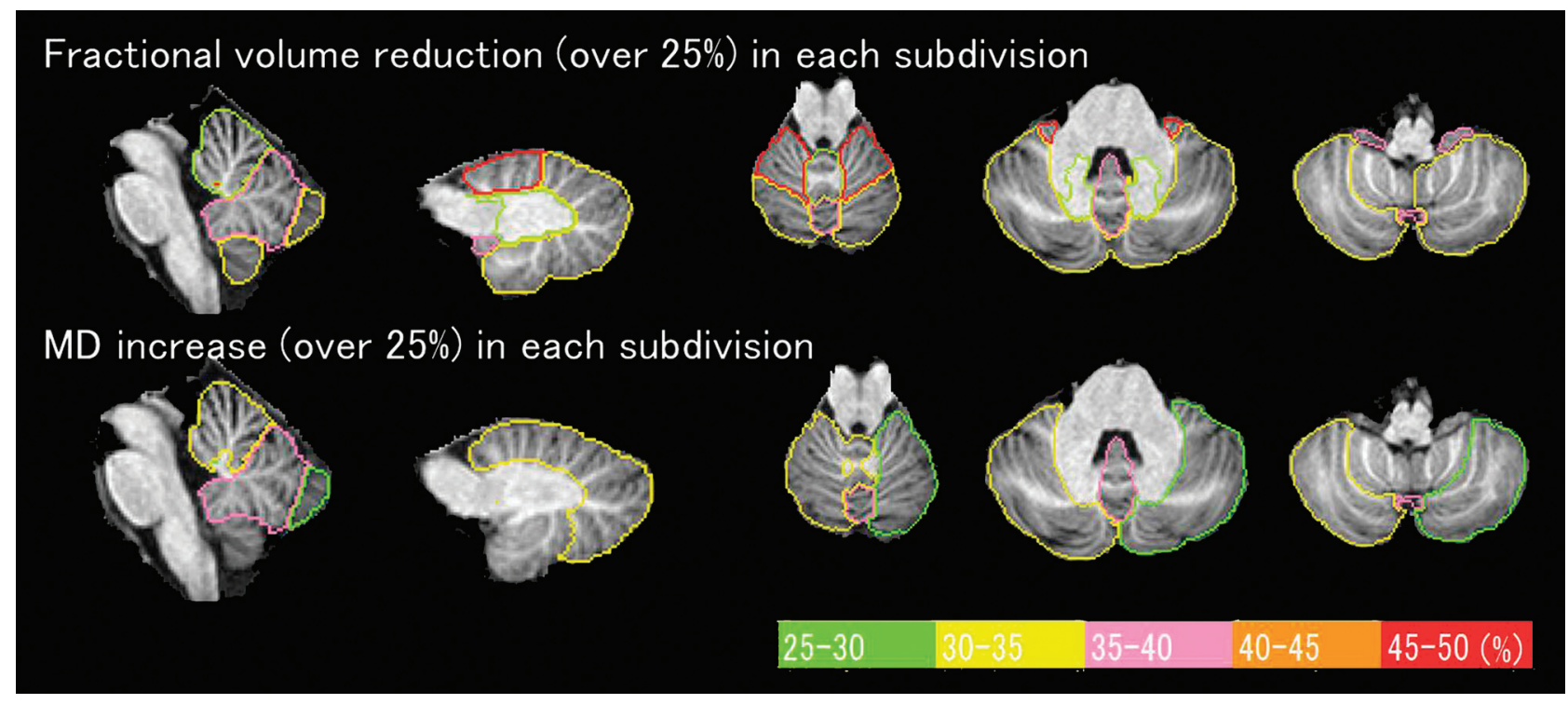

FIG 3. Percentage changes of fractional volume and MD in patients with SCA6 compared with controls (changes of $>25 \%$ are shown). The degrees of percentage change are presented in different colors. The changes of FA are $<25 \%$ and are not shown.

subscores was the static score and the fractional volume in the right flocculonodular lobe $(P<.01, r=-0.92)$. FA showed a significant correlation only with the limb score in the left posterior lobe and in the posterior vermis $(P<.05, r=-0.73)$. In the brain stem, the fractional volume was significantly decreased in all of the cerebellar peduncles $(P<.01$ to $<.05)$. MD was significantly increased in the SCP and the MCP $(P<.0001$ to $<.05)$. A significant increase of FA was seen in the MCP $(P<.05)$. The ICARS total score was significantly correlated with the fractional volume in the midbrain $(P<.05, r=-0.77)$, and the ICARS static score was significantly correlated with the SCP $(P<.05, r=$ $-0.71)$.

\section{Percentage Changes of the Data}

Percentage changes of the data in the patients with SCA6 compared with the controls are shown in Fig 3. This procedure clarified the difference in the results that depended on subdivisions by excluding the effects of the volume difference of each subdivision on the results of fractional volume.

Atrophy of the bilateral anterior lobes was the most prominent, followed by atrophy of the flocculonodular lobes and the posterior vermis. MD showed the largest increase in the posterior vermis. In terms of phylogenetic origins, atrophy was seen mainly in old regions, and increased MD was seen mainly in new regions. FA decreased or increased depending on the subdivisions, and these changes were $<25 \%$. The correlations between the percentage changes and ICARS scores were indistinguishable from the correlations between the raw data and ICARS scores.

\section{Correlations between Fractional Volume and MD}

To examine the partial volume effects, we investigated the correlations between the fractional volume and MD of each infratentorial subdivision. No significant correlation was seen except in the left posterior lobe in the controls $(P<.05, r=-0.74)$.

\section{DISCUSSION}

The localization of cerebellar functions has been under debate for more than a century. ${ }^{25}$ Although much of the cerebellar topography is still unmapped, it is known that phylogenetically, the cerebellum has 3 origins: the archicerebellum, the paleocerebellum, and the neocerebellum. ${ }^{8,9,12}$ The archicerebellum corresponds to the flocculonodular lobes and may include the tonsils as well. The archicerebellum is also referred to as the "vestibulocerebellum" because it is connected to the vestibular nuclei, which maintain equilibrium and eye-movement control. The paleocerebellum includes the vermis and the hemispheres near the midline or anterior region, and it is also called the "spinocerebellum" because of its spinal connections, which receive somatosensory information and control posture/gait and muscle tone. The neocerebellum is the lateral part of the hemispheres, with principally corticopontocerebellar connections. It is also called the "cerebrocerebellum" and is related to the planning of movements and speech.

The LDDMM used in this study enabled us to transform subject images to template images with high accuracy by using a single normalized subject (Johns Hopkins University-Montreal Neurological Institute template). ${ }^{20,22,23}$ With LDDMM, we analyzed the subjects' brain images on the basis of anatomic units, and automated segmentation was possible on all subjects' images (atlas-based analysis). ${ }^{24,26}$

Our results confirmed the significant correlation of fractional volume in both the cerebellum and the brain stem with the symptoms of patients with SCA6 (Table). MD was recognized as another parameter to detect cerebellar degeneration. Although it has been reported that cerebral degeneration can occur in patients with SCA6 ${ }^{11,27}$ this finding was not demonstrated in our study.

Our detailed investigation of the cerebellum revealed a significant decrease of fractional volume and a significant increase of $\mathrm{MD}$ in all subdivisions in the cerebellum in the patients with SCA6 (Fig 2). Further analysis of the percentage changes excluded the effects of volume differences among the subdivisions, and in 
turn, the atrophy of small subdivisions became apparent (Fig 3). This procedure excluded unknown factors that could affect the correlation between the ICARS scores and the data, as well as age. The fractional volume was decreased mainly in phylogenetically older regions of the cerebellum, including the archicerebellum and the paleocerebellum. The largest atrophy was seen in the bilateral anterior lobes of the paleocerebellum, followed by atrophy of the posterior vermis and the bilateral flocculonodular lobes. These new findings have not been made clear in previous studies. Pathologically, the most prominent degeneration was reported in the archicerebellum, and the resultant vertigo is a characteristic feature in patients with SCA6. ${ }^{11}$

Contrary to fractional volume, a large increase of $\mathrm{MD}$ was seen mainly in new regions of the cerebellum, including the paleocerebellum and the neocerebellum (Fig 3). These regions include the cerebellar cortex. MD represents the degree of diffusion independent of the orientation of the tissue. ${ }^{28,29}$ The increase of MD in new regions may thus be related to degeneration of the cerebellar cortex, but the correct interpretation of the results has not yet been established. MD often increases due to partial volume effects - that is, the contamination of CSF. ${ }^{30}$ However, we observed no significant correlation between fractional volume and MD in most subdivisions. In addition, the MD was increased in the deep structures in which partial volume effects should be absent or subtle. These findings suggest that MD reflects other phenomena, not only partial volume effects.

In the brain stem, all the cerebellar peduncles showed significant decreases of fractional volume. MD was significantly increased in the SCP and the MCP. FA was also increased in the MCP. The percentage changes were similar.

Several studies have investigated changes of volume in the brain stem, ${ }^{3,6}$ but they did not separate the cerebellar peduncles from the brain stem, or they investigated only the cerebellar peduncles by using manually placed regions of interest. ${ }^{15}$ The cerebellar peduncles are neural pathways connecting the cerebellum and the brain stem or the cerebrum, which in turn make the circuits in the cerebellum to control movement. ${ }^{8,9}$ DTI enabled us to separate the cerebellar peduncles from the brain stem, and it was revealed that the reported atrophy of the brain stem in patients with SCA6 may be due to atrophy of the cerebellar peduncles.

When Purkinje cells are damaged, inhibitory effects by their efferent fibers would be affected. ${ }^{31}$ This result explains the decrease of fractional volume and increased MD in the SCP in patients with SCA6 in the present study because the SCP is the main efferent pathway from the cerebellum. The cerebellar cortex receives afferent fibers mainly from the midbrain and the medulla via the MCP and the ICP. Afferent fibers might be also affected because atrophy or increased MD were seen in the MCP and the ICP.

A decrease in FA was seen only in limited subdivisions (Fig $2 C)$. Increased FA was seen in the MCP. FA was increased in the brain stem and in the deep structures, too. In patients with SCA6, the main affected region is the cerebellar cortex. ${ }^{32,33}$ The utility of FA as a parameter is unclear without direct white matter damage, though subsequent white matter degeneration can occur. Takahashi et $\mathrm{al}^{34}$ reported that folial white matter degeneration sec- ondary to cortical degeneration was observed in patients with SCA6, but deep white matter was preserved. In general, it is difficult to detect the changes of FA in the cortex due to its isotropic feature. $^{35,36}$

The cerebellum has a complex network, ${ }^{8}$ which indicates that it has isotropic features. As a certain fiber in a subdivision in the cerebellum degenerates, FA in the subdivision can increase because of more unified fiber orientations. Changes of FA are likely to be subtle in chronic lesions compared with acute lesions due to the recovery of FA. ${ }^{37,38}$ These reasons might explain the unexpected changes of FA in our results.

Similar to the percentage changes, some ICARS scores were well-correlated with the fractional volume mainly in older regions and with MD mainly in newer regions (Table). Reflecting this tendency, the ICARS total score showed strong correlations in fractional volume in the bilateral deep structures and the right flocculonodular lobe and in MD in the bilateral posterior lobes ( $r=0.73$ to \pm 0.87 ). Strong correlations in fractional volume in the bilateral deep structures are understandable because most infratentorial fibers pass through the corpus medullare. Atrophy of the deep structures has not been well-studied, but in the present study, it was nearly $30 \%$ compared with that in controls. Analogously, in the brain stem, the midbrain showed a significant correlation with the ICARS total score.

The ICARS static score showed significant correlations in the archicerebellum and paleocerebellum. The right flocculonodular lobe in particular showed a very strong correlation with fractional volume $(r=-0.92)$. The flocculonodular lobes receive fibers from the vestibular nuclei and play an important role in balance. ${ }^{10,11}$ Our results were consistent with this feature. A correlation between the ICARS static score and fractional volume was also seen in the SCP. The SCP is the main path of the efferent fibers from the cerebellum, and our results confirmed its importance.

The ICARS limb score showed significant correlations in the neocerebellum and the right deep structure because both are related to limb function. ${ }^{9}$ It is known that the cerebellar nuclei in the deep structures are also related to limb movement.

The ICARS dysarthria score showed significant correlations in the archicerebellum and in the left cerebellar hemisphere. It is known that development of language in the brain is asymmetric and that lesions of the superior paravermal region are commonly associated with dysarthria, ${ }^{9}$ and our results are consistent with this description. The correlation that we observed between the dysarthria score and the archicerebellum is a new finding.

There were some limitations in this study. First, the number of subjects was small. SCA6 is rare, but studies of larger numbers of subjects would add credibility to the present findings. Characteristic site-specific degeneration patterns in patients with SCA6 could be useful for differential diagnoses, but comparisons with patients with multiple system atrophy, other types of SCA, or secondary ataxia are necessary.

\section{CONCLUSIONS}

We found characteristic macro- and microstructural changes depending on phylogenetic regions of the infratentorial brain that strongly correlated with clinical symptoms in patients with SCA6. 


\section{ACKNOWLEDGMENTS}

We give special thanks to Shigeki Aoki for his assistance with the interpretation of the data.

Disclosures: Kanako Sato-RELATED: Grant: Japan Society for the Promotion of Science, Comments: My stay at Johns Hopkins University School of Medicine for this study was supported by the International Training program provided by Japan Society for the Promotion of Science; UNRELATED: Payment for Manuscript Preparation: University research institute for diseases of old age, Juntendo University, Comments: Assistance for writing the manuscript in English was supported. Sarah $\mathrm{H}$. Ying-RELATED: Grant: National Institutes of Health*; UNRELATED: Employment: Johns Hopkins University; Grants/Grants Pending: National Institutes of Health.* Kenichi Oishi-UNRELATED: Grants/Grants Pending: National Institutes of Health/ Eunice Kennedy Shriver National Institute of Child Health and Human Development (R01HD065955).* Michael I. Miller—RELATED: Grant: supported by standard grants from the National Institutes of Health; Board Membership: AnatomyWorks, Comments: Susumu Mori is the president; I hold equity in this company. Susumu MoriRELATED: Grant: National Institutes of Health,* Comments: ROIAG020012, R01NS084957, P41EB015909; UNRELATED: Patents (planned, pending or issued): There are issued and pending patents that are not related to the presented work*; Other: CEO of AnatomyWorks, a startup company. ${ }^{\star}$ Money paid to the institution.

\section{REFERENCES}

1. Taroni F, DiDonato S. Pathways to motor incoordination: the inherited ataxias. Nat Rev Neurosci 2004;5:641-55

2. Nagaoka U, Suzuki Y, Kawanami, T, et al. Regional differences in genetic subgroup frequency in hereditary cerebellar ataxia, and a morphometrical study of brain MR images in SCA1, MJD and SCA6. J Neurol Sci 1999;164:187-94

3. Eichler L, Bellenberg B, Hahn HK, et al. Quantitative assessment of brain stem and cerebellar atrophy in spinocerebellar ataxia types 3 and 6: impact on clinical status. AJNR Am J Neuroradiol 2011; 32:890-97

4. Jacobi H, Hauser TK, Giunti P, et al. Spinocerebellar ataxia types 1, 2, 3 and 6: the clinical spectrum of ataxia and morphometric brainstem and cerebellar findings. Cerebellum 2012;11:155-66

5. Yagishita A. Imaging Diagnosis of Neurological Diseases [in Japanese]. Tokyo, Japan: Shujunsya; 2011:42

6. Schulz JB, Borkert J, Wolf S, et al. Visualization, quantification and correlation of brain atrophy with clinical symptoms in spinocerebellar ataxia types 1, 3 and 6. Neuroimage 2010;49:158-68

7. Jung BC, Choi SI, Du AX, et al. Principal component analysis of high resolution MRI defines disease-specific mode of cerebellar neurodegeneration in SCA types 2 and 6. Cerebellum 2012;11;887-95

8. Eccles JC. Circuits in the cerebellar control of movement. Proc Natl Acad Sci U S A 1967;58:336-43

9. Grimaldi G, Manto M. Topography of cerebellar deficits in humans. Cerebellum 2012;11:336-51

10. Fulton JF, Dow RS. The cerebellum: a summary of functional localization. Yale J Biol Med 1937;10:89-119

11. Wang X, Wang H, Xia Y, et al. Spinocerebellar ataxia type 6: systematic patho-anatomical study reveals different phylogenetically defined regions of the cerebellum and neural pathways undergo different evolutions of the degenerative process. Neuropathology 2010;30:501-14

12. Dietrichs E. Clinical manifestation of focal cerebellar disease as related to the organization of neural pathways. Acta Neurol Scand 2008;117:6-11

13. Mandelli ML, De Simone T, Minati L, et al. Diffusion tensor imaging of spinocerebellar ataxias types 1 and 2. AJNR Am J Neuroradiol 2007;28:1996-2000

14. Prakash N, Hageman N, Hua X, et al. Patterns of fractional anisotropy changes in white matter of cerebellar peduncles distinguish spinocerebellar ataxia-1 from multiple system atrophy and other ataxia syndromes. Neuroimage 2009;47(suppl 2):T72-81

15. Ying SH, Landman BA, Chowdhury S, et al. Orthogonal diffusionweighted MRI measures distinguish region-specific degeneration in cerebellar ataxia subtypes. J Neurol 2009;256:1939-42

16. Du AX, Cuzzocreo JL, Landman BA, et al. Diffusion tensor imaging reveals disease-specific deep cerebellar nuclear changes in cerebellar degeneration. J Neurol 2010;257:1406-08

17. Trouillas $P$, Takayanagi $T$, Hallett $M$, et al. International Cooperative Ataxia Rating Scale for pharmacological assessment of the cerebellar syndrome: the Ataxia Neuropharmacology Committee of the World Federation of Neurology. J Neurol Sci 1997;145:205-11

18. Jones DK, Horsfield MA, Simmons A. Optimal strategies for measuring diffusion in anisotropic systems by magnetic resonance imaging. Magn Reson Med 1999;42:515-25

19. Mori S, Oishi K, Jiang H, et al. Stereotaxic white matter atlas based on diffusion tensor imaging in an ICBM template. Neuroimage 2008;40:570-82

20. Oishi K. Atlas-based whole brain analysis using large deformation diffeomorphic metric mapping: application to normal elderly and Alzheimer's disease participants. Neuroimage 2009;46:486-99

21. Woods RP, Grafton ST, Watson JD, et al. Automated image registration. II. Intersubject validation of linear and nonlinear models. J Comput Assist Tomogr 1998;22:153-65

22. Beg MF, Miller MI, Trouve A, et al. Computing large deformation metric mappings via geodesic flows of diffeomorphisms. Int J Comput Vis 2005;61:139-57

23. Miller MI, Trouve A, Younes L. On the metrics and Euler-Lagrange equations of computational anatomy. Annu Rev Biomed Eng 2002; 4:375-405

24. Miller MI, Beg MF, Ceritoglu C, et al. Increasing the power of functional maps of the medial temporal lobe by using large deformation diffeomorphic metric mapping. Proc Natl Acad Sci U S A 2005; 102:9685-90

25. Buckner RL, Krienen FM, Castellanos A, et al. The organization of the human cerebellum estimated by intrinsic functional connectivity. J Neurophysiol 2011;106:2322-45

26. Faria AV, Zhang J, Oishi K, et al. Atlas-based analysis of neurodevelopment from infancy to adulthood using diffusion tensor imaging and applications for automated abnormality detection. Neuroimage 2010;52:415-28

27. Seidel K, Siswanto S, Brunt ER, et al. Brain pathology of spinocerebellar ataxias. Acta Neuropathol 2012:124:1-21

28. Basser PJ, Jones DK. Diffusion-tensor MRI: theory, experimental design and data analysis - a technical review. NMR Biomed 2002; 15:456-67

29. Le Bihan D, Turner R, Douek P, et al. Diffusion MR imaging: clinical applications. AJR Am J Roentgenol 1992;159:591-99

30. Kang XJ, Herron TJ, Turken U, et al. Diffusion properties of cortical and pericortical tissue: regional variations, reliability and methodological issues. Magn Reson Imaging 2012;30:1111-22

31. Iwata NK, Ugawa Y. The effects of cerebellar stimulation on the motor cortical excitability in neurological disorders: a review. Cerebellum 2005;4:218-23

32. Sasaki H, Kojima H, Yabe I, et al. Neuropathological and molecular studies of spinocerebellar ataxia type 6 (SCA6). Acta Neuropathol 1998;95:199-204

33. Yang Q, Hashizume Y, Yoshida M, et al. Morphological Purkinje cell changes in spinocerebellar ataxia type 6. Acta Neuropathol 2000; 100:371-76

34. Takahashi H, Ikeuchi T, Honma Y, et al. Autosomal dominant cerebellar ataxia (SCA6): clinical, genetic and neuropathological study in a family. Acta Neuropathol 1998;95:333-37

35. Le Bihan D, van Zijl P. From the diffusion coefficient to the diffusion tensor. NMR Biomed 2002;15:431-34

36. Mori S, Zhang J. Principles of diffusion tensor imaging and its applications to basic neuroscience research. Neuron 2006;51:527-39

37. Tievsky AL, Ptak T, Farkas J. Investigation of apparent diffusion coefficient and diffusion tensor anisotropy in acute and chronic multiple sclerosis lesions. AJNR Am J Neuroradiol 1999;20:1491-99

38. Takagi T, Nakamura M, Yamada M, et al. Visualization of peripheral nerve degeneration and regeneration: monitoring with diffusion tensor tractography. Neuroimage 2009;44:884-92 